\title{
Index autorum ad Vol. 14
}

Adler, P. 338

Andrade, J. D. 133

Andresen, E. 319

Arndt-Hanser, A. 298

Åkesson, H. O. 265

Bähr, M.-L. 15

Baitsch, H. 4, 25, 366

Beam, A. G. 41, 52

Beçak, M. L. 133

Beçak, W. 133

Beckman, L. 159, 286, 309

Benerecetti-Santachiara, A. S. 36

Bernhard, W. 298

Das, S. R. 168

Defrise-Gussenhoven, E. 351

Dichgans, I. 240

Dodinval, P. 327

Dodinval-Versie, J. 327

Edwards, J. H. 76

Forssman, H. 265

Glen-Bott, A. M. 52

Goedde, H. W. 366

Grivea, M. 159

Harris, H. 52

Heiken, A. 57

Ikin, W. 57

Johnson, F. M. 309

Kirk, R. L. 41

Kornhuber, H. H. 240

Kurito, T. 67

Lander, E. 265

Leek, I. 76

Leon, N. 143

Malchair, R. 327 Mermod, L. E. 25 Minet, P. L. 186 Modiano, G. 36 Mohr, J. 125 Moureau, P. 327 Mukherjee, D. P. 168 Nilsson, S. E. 97 Parker, W.C. 41, 52 Parsons, P. A. 201 Pinkerton, F. J. 25 Polczer, M. G. 338 Quelce-Salgado, A. 63 Record, R. G. 76 Remy, K. 4 Renkonen, K. O. 177 Ritter, H. 4, 15, 25 Rivat, L. 4, 15, 25, 298 Robson, E. B. 52 Ropartz, C. 4, 15, 25, 298 Rousseau, P.-Y. 4, 15, 25, 298 Sakai, R. K. 309 Saldanha, D. H. 143 Salzano, F. M. 212 
Schmidt, B. J. 143 Sergovich, F. R. 251 Soltan, H. C. 251 Tonomura, A. 67 Walter, H. 298

Went, L. N. 220 Whittaker, M. 281 Wiens, R. G. 251 Woods, J. L. 309 